\title{
PERINATAL RISK FACTORS ASSOCIATED WITH CEREBRAL PALSY IN CHILDREN BORN IN ODESA OBLAST REGION (UKRAINE)
}

\author{
Posokhova S.P., Kucherenko O.U. Odesa National Medical University
}

The etiology of cerebral palsy (CP) is very diverse and multifactorial including prenatal, natal, and postnatal factors Studies have reported that the prevalence of cerebral palsy may vary between 1.5 and 3.0 per 1000 live births. Several hypotheses have been proposed to explain the origins of $\mathrm{CP}$ in very preterm babies.

It may be the result of an ischemic insult in utero leading to both preterm birth anddamage to the white matter. The immature babies are particularly vulnerable to cerebral hemorrhage and ischemia. Neonatal factors such as: seizures, prolonged ventilation, intraventricular hemorrhage, periventricular leukomalacia, bronchopulmonary dysplasia, sepsis.

Methods. The aim of this study was to identify antenatal, intrapartum and neonatal risk factors for cerebral palsy. Antenatal, intrapartum, and neonatal events were compared between 100 children with $\mathrm{CP}$ and 100 controls in a retrospective case-control method. Antenatal, intrapartum and neonatal factors were expressed as odds ratios and $95 \%$ confidence intervals.

Results. Major risk factors found in this study were history of spontaneous abortions in $28(28 \%)$, anemia during pregnancy in $35(35 \%)$, hypertension in $12(12 \%)$, obesity of $2-3$ degrees in $18 \%$ and history of infection in $8 \%$ mothers. In total, 44 $(44 \%)$ children were born preterm and $10(10 \%)$ were born in multiple births. Infants born preterm had a highly increased risk for CP, and constituted 44\% of all cases; OR 21.8 (95\% CI 29$39)$ in weeks $26-28$, OR 29 (95\% CI 32-

42) in weeks 29-30, OR 42 (95\% CI 24-70) in weeks 31-32, and OR 44.7 (95\% CI 34-77) in weeks 33-34. Other factors associated with $\mathrm{CP}$ were being small or large for gestational age at birth, intrauterine growth restriction (IUGR) OR 7.4 (95\% CI 2.4-18.5), abruptio placentae (OR 8.6, 95\% CI 5.613.3), preeclampsia (OR 42,95\% CI 2.4-7.7), being a twin (OR 25.5, 95\% CI 1.5-45.5), smoking (OR 4.1, 95\% CI 1.115). In term infants, low Apgar scores were associated with a high risk for CP; OR 53.2 (95\% CI 31-89) at score 6 at 5 minutes, OR 104 (95\% CI 6.2-172) at score 3. Other factors associated with $\mathrm{CP}$ in term infants were breech presentation at vaginal birth (OR 3.8, 95\% CI 2.4-10.4), instrumental delivery (OR 2.9, 95\% CI 1.6-5.3), and emergency cesarean delivery (OR 2.5, 95\% CI 1.6-6.2).

The most frequent risk factors in the postnatal period were high fever in $12 \%$, convulsion in $34 \%$, intraventricular hemorrhage in $21 \%$, hypoxic-ischemic encephalopathy in $28 \%$ and jaundice in $16 \%$ of newborns. Respiratory distress syndrome, prolonged ventilation were in $25 \%$ of newborns. Severe cranial ultrasound abnormality in $45 \%$ newborns were associated with an increased risk of $\mathrm{CP}$ in the neonatal period.
Table 1. Risk factors for CP

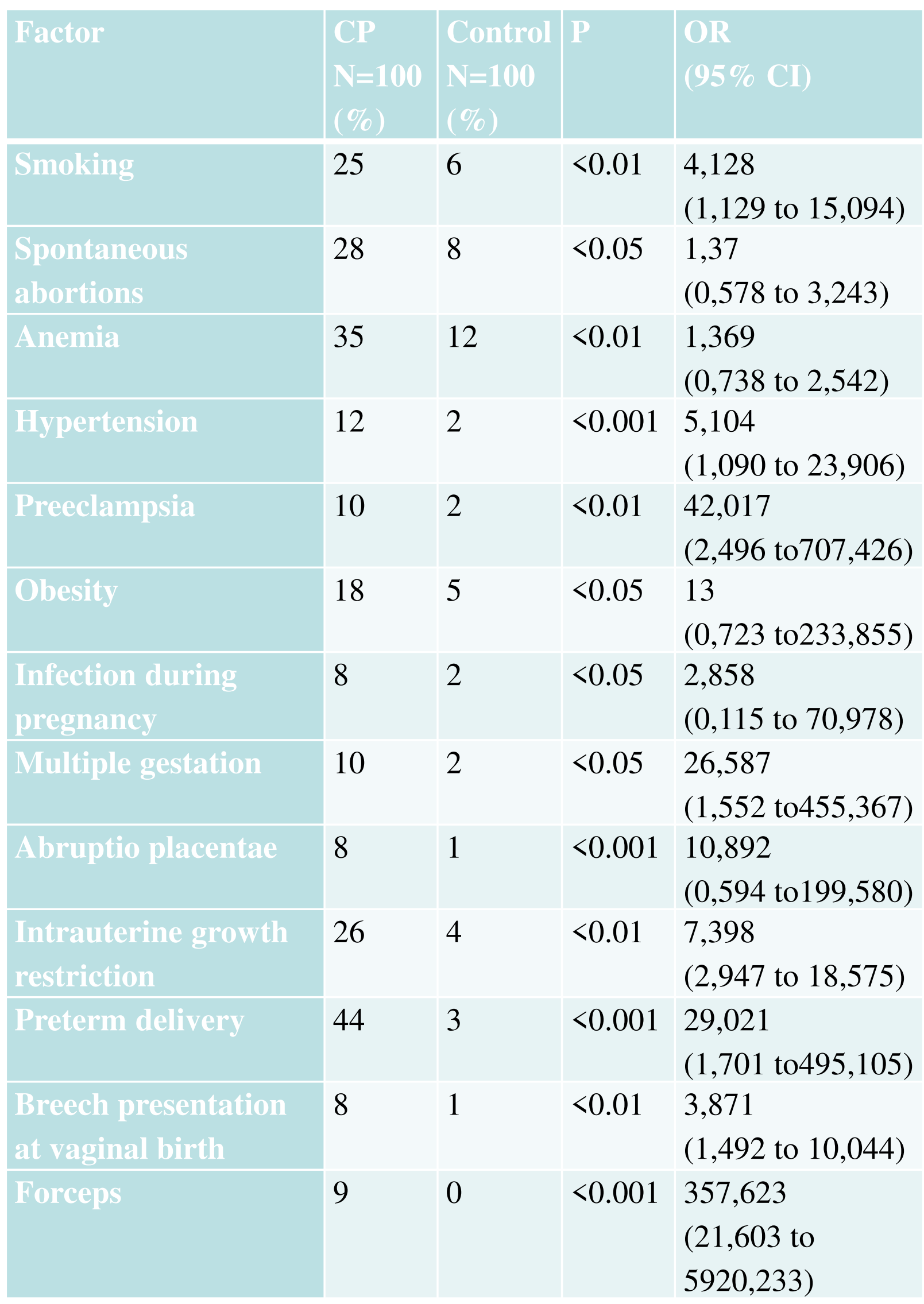

Weight and Apgar score

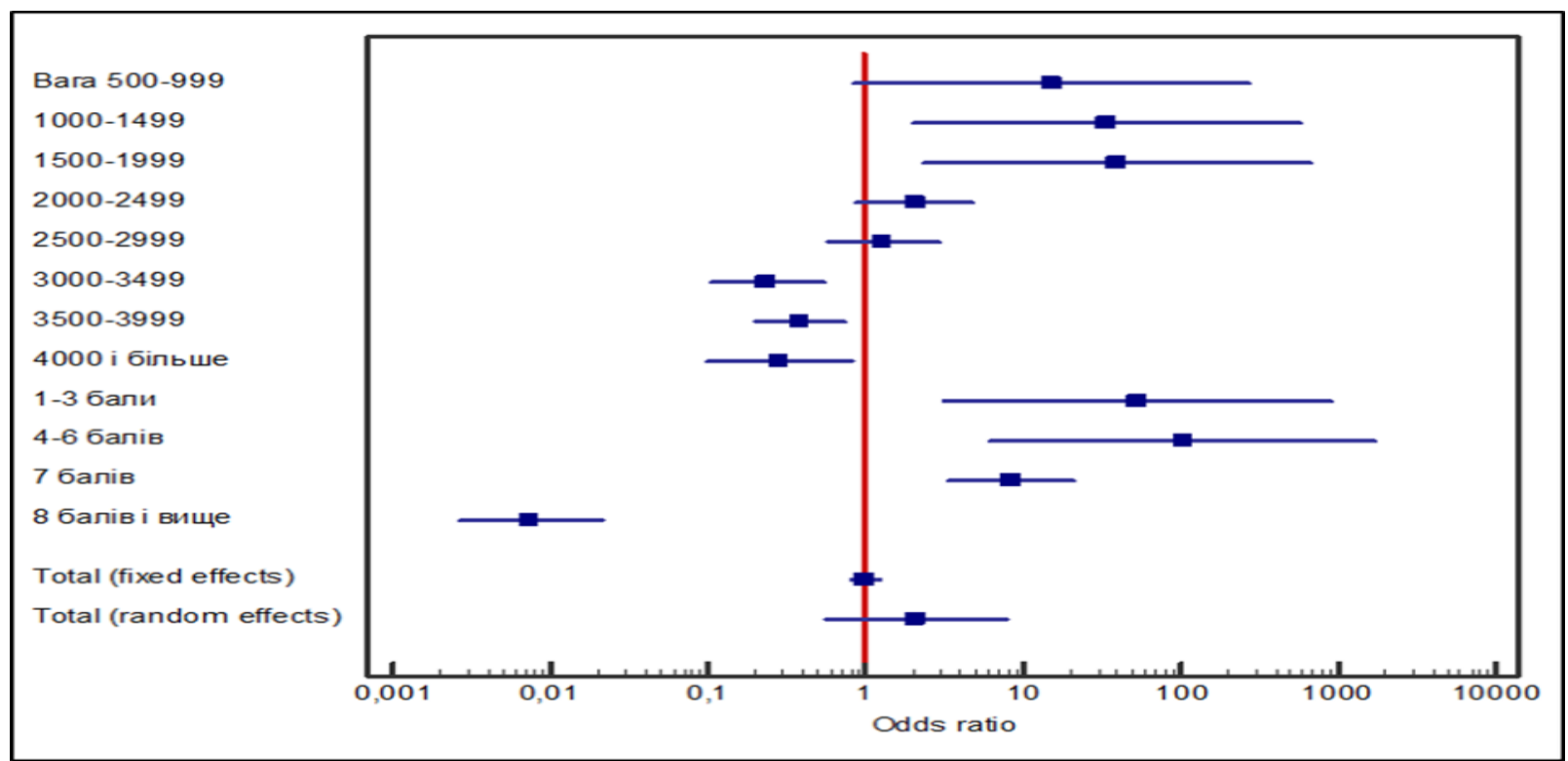

Conclusions: Our findings confirm that several antenatal factors as smoking, preeclampsia, obesity, anemia, IUGR; intrapartum factors as abruption placenta, preterm birth, breech presentation, low Apgar scores and neonatal risk factors as convulsion, intraventricular hemorrhage, hypoxic-ischemic encephalopathy, jaundice, respiratory distress syndrome, prolonged ventilation in babies are responsible for the etiology of cerebral palsy.

Key words: cerebral palsy; perinatal and postnatal risk factors. 